\title{
Deep tumor imaging by upconversion nanoparticles
}

\author{
Nataly Sholina ${ }^{1,2,3,}$, , Polina Demina ${ }^{3}$, Dmitry Khochenkov ${ }^{1,3}$, Alla Generalova ${ }^{3,4}$, Andrey \\ Nechaev $^{5}$, and Eugeny Khaydukov ${ }^{2,3}$ \\ ${ }^{1}$ N. N. Blokhin Russian Cancer Research Center, Moscow 115478, Russia. \\ ${ }^{2}$ I. M. Sechenov First Moscow State Medical University, Moscow 119991, Russia. \\ ${ }^{3}$ Federal Scientific Research Center Crystallography and Photonics RAS, Moscow 119333, Russia \\ ${ }^{4}$ Shemyakin-Ovchinnikov Institute of Bioorganic Chemistry RAS, Moscow 117997, Russia \\ ${ }^{5}$ Institute of Fine Chemical Technologies, Moscow Technol. University, Moscow 119571, Russia
}

\begin{abstract}
In this work are shown the prospects of using upconversion nanoparticles (UCNPs) as markers for contrast optical imaging of a tumor. For using nanoparticles for biomedical purposes is implemented a technique for coating nanoparticles with polymers, such as PEG and PSA. This approach provides low non-specific adsorption, which prolongs the circulation of UCNPs in mouse bearing Lewis Lung Cancer (LLC) up to 10 hours. These properties allow nanoparticles to quickly accumulate in the tumor. Effective delivery of particles with different polymer coatings in the tumor is demonstrated with the help of an epiluminescent imaging system.
\end{abstract}

Today one of the topical directions in modern medical diagnostics is the creation of nanoprobes aimed at pathological targets based on photoluminescent nanoparticles possessing high photo- and chemical stability and their application in real time. Such nanoparticles are able to specifically label tumor tissues and ensure their visualization in real time [1].

Upconversion nanoparticles (UCNPs) are a special class of luminescent nanoparticles with a specific property - the ability to conversion low-energy photons into photons of higher energy. UCNPs are inorganic nanocrystals (NaYF4) doped with lanthanide ions. Their photophysical properties provide a bright and long-term visualization of the structures marked by them in the depth of biological tissues. UCNPs are characterized by photostability, low toxicity, and their surface, allows the creation of conjugates with targeting modules. The wavelength of excitation and photoluminescence UCNPs falls into the so-called "window of biotissue transparency", which allows them to be visualized in biological tissues from a depth of several centimetre [2]. They have chemically active functional groups on the surface, which is important for obtaining targeted drugs, and their optimal size determines the predominant accumulation of nanoparticles in the tumor tissue due to the morphological features of its vascular system due to the EPR-effect. It is important to note that one of the main difficulties in the targeted delivery of nanoparticles is

*Corresponding author: sholinanv@gmail.com 
their insufficient circulation time in the blood and significant absorption by macrophages, which reduces their accumulation in the tumor. To improve the targeted delivery and reduce non-specific absorption by macrophages, we modified their surface with hydrophilic biocompatible coatings based on polyethylene glycol (PEG) and polysialic acid (PSA), which increased the circulation time in the bloodstream from several minutes to 10 hours (in the case with PSA) (Fig.1A).

In order to visualize the tumor with the UCNPs with coatings, we performed a study on C57Bl/6 mice bearing Lewis lung cancer. To visualize the fluorescent signal, an imager of small animals DVS-02 (Institute of Photon Technologies of the RAS (Troitsk, Moscow)) was used. UCNPs at a concentration of $800 \mu \mathrm{g} / \mathrm{ml}$ in physiological saline was administered intravenously to the animals in a volume of $0.15 \mathrm{ml}$. After the administration of UCNPs, a series of epiluminescent images and spectral characteristics of the tumor were obtained to study the accumulation of nanoparticles in the tumor.

According to the obtained data, an increase in the photoluminescent signal from the tumor begins after 1 minute, with an increase in contrast as compared to the surrounding tissues. In the range of 2-5 minutes, there is a slight decrease in the signal from the tumor, which is supposed to be due to the redistribution of nanoparticles with circulating blood. Five minutes after the administration of UCNPs, the signal grows in the tumor (due to the accumulation and retention of circulating UCNPs), reaching a maximum after 60 min after administration (Fig.1B).
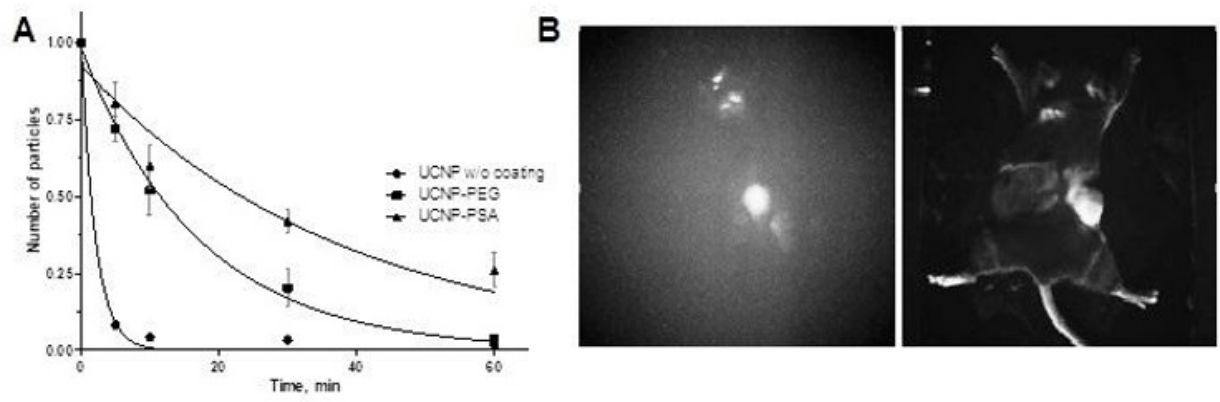

Fig. 1. (A) Normalized dependence of the UCNPs without biocompatible coating, and UCNPs surface modified with PEG and PSA vs. blood circulation time. (B) Epiluminescent images of C57B1/6 mice bearing Lewis lung cancer obtained after 60 min UCNP-PSA injection (left) and general view (right).

The recorded photoluminescence signal was maintained for 24 hours after administration of UCNPs. The strongest photoluminescent signal was recorded from UCNPs coated with PSA, which is associated with a more significant accumulation of particles in the tumor. The smallest signal was recorded from hydrophilic UCNPs without a biocompatible coating. The conducted studies show the prospects of using nanoparticles of modified PSA for marking and intravital imaging of tumors. This technology can be in demand for intraoperative assessment of the boundaries of surgical intervention. This work was supported by Russian Science Foundation (RSF) grant 16-13-10528.

\section{References}

1. A. Nadort, V.K.A. Sreenivasan, Z. Song, E.A. Grebenik, A.V. Nechaev, V.A. Semchishen, V.Ya. Panchenko, A.V. Zvyagin, PLoS ONE 8, e63292 (2013)

2. B.J. Tromberg, N. Shah, R. Lanning, A. Cerussi, J. Espinoza, T. Pham, J. Butler, Neoplasia 2, 26 (2000) 